\title{
ASSOCIATIONS OF SUBJECTIVE SLEEP QUALITY WITH DEPRESSION SCORE, ANXIETY, PHYSICAL SYMPTOMS AND SLEEP ONSET LATENCY IN STUDENTS
}

\author{
Christoph Augner \\ IGGMB - Research Institute for Basic and Frontier Questions of Medicine and Biotechnology, Health Research Institute, University Clinics of \\ the Paracelsus Medical University, Salzburg, Austria
}

\begin{abstract}
SUMMARY
Objective: Sleep quality is strongly associated with parameters of mental and physical health and therefore a major public health issue. We wanted to evaluate this association in young and healthy students by a survey. Further, we aimed to detect the relevance of sleep onset latency and sleep duration for sleep quality.

Methods: A group of young nursing and technical students was surveyed $(\mathrm{N}=196)$ with the objective to measure subjective sleep quality, sleep onset latency, sleep duration, depression score, physical symptoms, trait-anxiety, and pathological eating behaviours.

Results: Subjective sleep quality was strongly negative correlated with depression score (Pearson's $r=-0.57$ ), physical symptoms ( $r=-0.51$ ) and trait-anxiety $(r=-0.54)(p<0.001$ for all three). Subjective sleep quality's association with sleep onset latency was stronger than with sleep duration. Further, high depression score (odds ratio $\mathrm{OR}=3.90 ; 95 \%$ confidence interval $\mathrm{Cl}=1.88-8.06$ ) and long sleep onset latency (OR=3.56; $95 \% \mathrm{Cl}=1.65-7.69$ ) were the best predictors of poor subjective sleep quality.

Conclusions: This study supports evidence that links physical and mental symptoms with poor sleep quality. Especially important is the fact that we found this connection in young and basically healthy adults. Taking into account that poor sleep quality has major negative long term impact on health, prevention programmes should focus especially on the association between depressive symptoms and subjective sleep quality that is significantly influenced by sleep onset latency.
\end{abstract}

Key words: sleep, mental health, public health, depression

Address for correspondence: Ch. Augner, IGGMB - Research Institute for Basic and Frontier Questions of Medicine and Biotechnology, Health Research Institute, University Clinics of the Paracelsus Medical University, Salzburg, Austria. E-mail: c.augner@salk.at

\section{INTRODUCTION}

Sleep quality is an important factor for health. There is evidence that insufficient sleep can lead to more accidents (1). Poor sleep quality has major impacts on physical health in the long run. A significant increase of heart disease risk may even result in death (2). Furthermore, sleep quality is often discussed in context with mental health. Evidence supports the hypothesis that poor sleep quality is associated with decreased mental health and well-being (3-8).

Psychiatric patients frequently experience sleep problems and poor sleep quality (4). There is evidence that sleep quality is associated with poor mental health status in children and adolescents: onset and lasting of poor mental health was significantly correlated with sleep disturbances in a group of about 500 Japanese junior high school students (6). Hayashino et al. (8) found in a population-based survey, that the number of comorbid conditions was significantly associated with sleep quality and depression. Le Blanc et al. (3) found correlations between insomnia symptoms and lower quality of life, higher scores in depression, anxiety, stress perception and other variables.
Due to the major relevance of sleep quality and health, the objective of this study was to evaluate the association between subjective sleep quality and health parameters in basically healthy students. This group of young adults was neglected in recent studies that focused mainly on adolescents, clinical or representative groups. Sleep duration was included as part of sleep quality most of the time, surprisingly sleep onset latency played a subordinate role in sleep quality measurement. Therefore, we aimed to detect if self rated sleep onset latency and sleep duration affected subjective sleep quality.

\section{METHODS}

Data analysed for this paper were acquired in a survey related to mobile phone behaviours. 196 ( $76.53 \%$ female and $23.47 \%$ male; mean age was $20.05, \mathrm{SD}=3.21$ ) students of three different nurse's training schools in Upper Austria, and one technical college in Salzburg, Austria, were included in this data analysis. Participants had to obtain written informed consent, procedures were in accordance with the ethical guideline of expanded 
Helsinki Declaration (9) and those suggested by the American Psychological Association (10).

In addition to demographic data we assessed sleep quality by a self created 3-item scale (e.g. How often during the past 2 weeks did you feel drowsy or sleepy during the day?). Response categories ranged from 0 (never) to 4 (very often). Reliability was sufficient (Cronbach's Alpha >0.50). Participants had to rate their average sleep duration and sleep onset latency during the last two weeks.

Further we used a self administered symptom checklist including ten very common physical symptoms (e.g. headache, back pain, nausea, fatigue etc.). Participants had to rate the frequency they had experienced each symptom during the last two weeks $(0$, never to 4 , very often; Cronbach's Alpha $=0.72$ ).

We used standardized questionnaires to measure trait anxiety (State-Trait Anxiety Inventory; STAI) (11) and to screen depressive symptoms (WHO-5 well being questionnaire; (12). Possible pathological eating behaviours were assessed by the SCOFF questionnaire consisting of five "yes"/"no"questions (13).

Statistics. Data were analysed by correlation analysis and logistic regression model (PASW Statistics 17).

Table 1. Correlation analysis between "Subjective sleep quality" and possible predictors

\begin{tabular}{|l|c|c|}
\hline \multirow{2}{*}{} & \multicolumn{2}{|c|}{ Subjective sleep quality } \\
\cline { 2 - 3 } & $\mathbf{r}$ & $\mathbf{p}$ \\
\hline Sleep onset latency & -0.35 & $<0.001$ \\
\hline Sleep duration & 0.20 & 0.005 \\
\hline Symptom score & -0.51 & $<0.001$ \\
\hline Depression score & -0.57 & $<0.001$ \\
\hline Trait anxiety score & -0.54 & $<0.001$ \\
\hline Pathological eating & -0.28 & $<0.001$ \\
\hline
\end{tabular}

$r=$ Pearson's correlation coefficient, $p=l e v e l$ of significance was defined as $(p<0.05)$.

Please note: High "subjective sleep quality" score indicates higher sleep quality.

\section{RESULTS}

As correlation analysis in Table 1 shows, subjective sleep quality is significantly associated with parameters of mental and physical health. There was a higher correlation between sleep onset latency and subjective sleep quality than with sleep duration. In further analysis, a regression model including gender was calculated (Table 2). Therefore continuous variables were dichotomized. For trait anxiety score (STAI), depression score (WHO-5) and symptom score we used median as a cut off point for high vs. low. Cut off point for sleep onset latency's was 20 minutes (till 20 minutes for short and $>20$ minutes for long), and 7 hours for sleep duration ( $<7$ hours for short and 7 or more hours for long). Pathological eating was defined as $>1$ yes answers in SCOFF as suggested by Hölling and Schlack (13). Best predictors for poor subjective sleep quality were high depression score and long sleep onset latency. Sleep duration and pathological eating behaviour were no significant predictors for poor subjective sleep quality (Table 2).

\section{DISCUSSION}

The present study supports previous results which suggested associations between poor sleep quality and parameters of mental and physical health. Self rating of sleep quality is not objective, but it represents what people actually experience and is therefore commonly used (14). Results show significant increased risk of poor sleep experience for persons with high depression score $(\mathrm{OR}=3.90, \mathrm{p}<0.001)$. Results also underline the importance of trait anxiety and symptoms for sleep quality.

Significant negative correlations between sleep quality and depression were also found by Hayashino et al. (8) and LeBlanc et al. (3) in general population samples. Significant negative relationships between sleep quality and depression as well as anxiety were detected by Mayers et al. (7) in a clinical sample. Kaneita et al. (6) reported positive associations between sleep disturbances and general poor mental health status in Japanese high school students.

We also included pathological eating behaviour in our analyses and did find significant correlations but no significant effect in

Table 2. Multivariate Logistic regression model: Predictors for "poor subjective sleep quality"

\begin{tabular}{|l|c|c|c|}
\hline \multirow{2}{*}{} & \multicolumn{2}{|c|}{ Poor subjective sleeping quality } & p \\
\cline { 2 - 4 } & OR & $95 \% \mathrm{Cl}$ & 0.144 \\
\hline Male gender & 0.51 & {$[0.21-1.26]$} & 0.001 \\
\hline Sleep onset latency >20 minutes & 3.56 & {$[1.65-7.69]$} & 0.134 \\
\hline Sleep duration <7 hours & 1.84 & {$[0.83-4.08]$} & 0.044 \\
\hline High Symptom score & 2.23 & {$[1.02-4.86]$} & $<0.001$ \\
\hline High Depression score & 3.90 & {$[1.88-8.06]$} & 0.020 \\
\hline High Trait anxiety score & 2.52 & {$[1.15-5.49]$} & 0.556 \\
\hline Pathological eating >1 & 1.27 & {$[0.58-2.80]$} & \\
\hline
\end{tabular}

OR=Odds Ratio, $\mathrm{Cl}=$ confidence interval, $\mathrm{p}=$ level of significance was defined as $(\mathrm{p}<0.05)$. Pathological eating was defined as SCOFF score $>1$ as suggested by Hölling and Schlack (13).

Please note: High "poor subjective sleep quality" indicates low sleep quality. 
regression model. Algul et al. (5) reported associations of poor sleep quality with obesity. Despite the fact that pathological eating may not be strongly associated with body mass index, we would also suppose that depression moderates the possible effect of obesity on sleep quality. There are research efforts that focus on the relationship of nutrition and sleep quality. Weiss et al. (15), for example, found that adolescents that slept less than 8 hours consumed higher proportion of calories from fats and lower proportion of calories from carbohydrates. Although these findings are important, sleep quality should always be seen in the context of mental health parameters, especially of depressive symptoms.

Research uses sleep duration as an important indicator for sleep quality $(1,15)$. Our results confirmed this findings but it was also found that sleep onset latency is even more important for subjective sleep quality. Difficulties to fall asleep were recently reported to have a prevalence of 7 to 16 percent in the general population while teenagers and young adults around 20 years are more commonly affected (16).

One of limitations of our study was the high female proportion in the sample $(76.53 \%)$, that can be attributed to the fact that most of the survey was done in nursing schools. Nevertheless, our study shows the relevance of mental health's and well-being's association with sleep quality, even in young and basically healthy individuals. For prevention purposes it is important to be aware of this association. When we talk about depressive symptoms, we should also focus on the increase of sleep quality. Improving sleep quality by learning functional methods for sleep hygiene and relaxation methods could even prevent depressive symptoms.

\section{Statement on conflict of interest}

None declared.

\section{REFERENCES}

1. Lombardi DA, Folkard S, Willetts JL, Smith GS. Daily sleep, weekly working hours, and risk of work-related injury: US National Health Interview Survey (2004-2008). Chronobiol Int. 2010 Jul;27(5):1013-30.

2. Schwartz S, McDowell Anderson W, Cole SR, Cornoni-Huntley J, Hays JC, Blazer D. Insomnia and heart disease: a review of epidemiologic studies. J Psychosom Res. 1999 Oct;47(4):313-33.
3. LeBlanc M, Beaulieu-Bonneau S, Mérette C, Savard J, Ivers H, Morin CM. Psychological and health-related quality of life factors associated with insomnia in a population-based sample. J Psychosom Res. 2007 Aug;63(2):157-66.

4. de Niet GJ, Tiemens BG, Lendemeijer HH, Hutschemaekers GJ. Perceived sleep quality of psychiatric patients. J Psychiatr Ment Health Nurs. 2008 Aug;15(6):465-70.

5. Algul A, Ates MA, Semiz UB, Basoglu C, Ebrinc S, Gecici O, et al. Evaluation of general psychopathology, subjective sleep quality, and health-related quality of life in patients with obesity. Int J Psychiatry Med. 2009;39(3):297-312.

6. Kaneita Y, Yokoyama E, Harano S, Tamaki T, Suzuki H, Munezawa T, et al. Associations between sleep disturbance and mental health status: a longitudinal study of Japanese junior high school students. Sleep Med. 2009 Aug;10(7):780-6.

7. Mayers AG, Grabau EA, Campbell C, Baldwin DS. Subjective sleep, depression and anxiety: inter-relationships in a non-clinical sample. Hum Psychopharmacol. 2009 Aug;24(6):495-501.

8. Hayashino Y, Yamazaki S, Takegami M, Nakayama T, Sokejima S, Fukuhara S. Association between number of comorbid conditions, depression, and sleep quality using the Pittsburgh Sleep Quality Index: results from a population-based survey. Sleep Med. 2010 Apr;11(4):366-71.

9. World Medical Association declaration of Helsinki. Recommendations guiding physicians in biomedical research involving human subjects. JAMA. 1997 Mar 19;277(11):925-6.

10. American Psychological Association. Ethical principles of psychologists and code of conduct [Internet]. Washington: American Psychological Association; 2002 [cited 2009 Jun 9]. Available from: http://www.apa. org/ethics/.

11. Laux L, Glanzmann P, Schaffner P, Spielberger CD. The State-traitanxiety questionnaire. Goettingen: Hogrefe; 1970. (In German.)

12. Löwe B, Spitzer RL, Gräfe K, Kroenke K, Quenter A, Zipfel S, et al. Comparative validity of three screening questionnaires for DSM-IV depressive disorders and physicians' diagnoses. J Affect Disord. 2004 Feb;78(2):131-40.

13. Hölling H, Schlack R. Eating disorders in children and adolescents. First results of the German Health Interview and Examination Survey for Children and Adolescents (KiGGS). Bundesgesundheitsblatt Gesundheitsforschung Gesundheitsschutz. 2007 May-Jun;50(5-6):794-9. (In German.)

14. Vangelova KK. Stress and fatigue in sound engineers: the effect of broadcasting in a life show and shift work. Cent Eur J Public Health. 2008 Jun; 16(2):87-91.

15. Weiss A, Xu F, Storfer-Isser A, Thomas A, Ievers-Landis CE, Redline S. The association of sleep duration with adolescents' fat and carbohydrate consumption. Sleep. 2010 Sep 1;33(9):1201-9.

16. Martinez D, Lenz Mdo C. Circadian rhythm sleep disorders. Indian J Med Res. 2010 Feb;131:141-9.

Received October 21, 2010 Accepted in revised form March 4, 2011 